\title{
Schlussfolgerungen und Ausblick
}

Security-Angriffe auf Verkehrssysteme können - gewollt oder ungewollt - zu negativen Auswirkungen auf die Betriebssicherheit (Safety) führen. Aus Sicht der Betriebssicherheit sind Maßnahmen zur Gewährleistung der Angriffssicherheit (Security) dann notwendig, wenn ein Risiko im Sinne der Betriebssicherheit aufgrund einer gezielten Manipulation so hoch ist, dass die Kritische Verkehrsinfrastruktur nicht mehr die erwartbare Betriebssicherheit bietet. Bei der Implementierung von Angriffs- und Betriebssicherheit sollten Synergien genutzt und Widersprüche vermieden werden. Dies wird durch die Synchronisation der Aktivitäten zwischen der Entwicklung funktional sicherer Systeme mit der Entwicklung angriffssicherer Systeme an den relevanten Schnittstellen im Lebenszyklus erreicht (Security Engineering). Gleichzeitig muss die Einhaltung der definierten Security-Eigenschaften nachgewiesen werden (Security Testing).

Da die Bedrohungen aus dem Internet einem stetigen Wandel unterliegen, ist das Security Management eine kontinuierliche Aufgabe des Betreibers einer Kritischen Verkehrsinfrastruktur. Nur so wird das erforderliche Schutzniveau erreicht und der sichere und ordnungsgemäße Betrieb aufrechterhalten. Insofern sind neben den technischen und physischen Schutzmaßnahmen auch organisatorische Vorkehrungen unerlässlich. Nur eine Organisation mit stabilen auf IT-Sicherheit bezogenen Prozessen wird in der Lage sein, die IT-Sicherheit dauerhaft aufrecht zu erhalten.

Die Herausforderung wird zukünftig sein, den unbestimmten Rechtsbegriff der ,angemessenen Maßnahmen“ pragmatisch zu definieren. Getreu der Devise „so viel wie nötig, so wenig wie möglich“ darf die Wettbewerbsfähigkeit insbesondere öffentlicher Verkehrssysteme im intermodalen Wettbewerb nicht durch überzogene Schutzmaßnamen negativ beeinträchtigt werden. 